\title{
Diode breakdown related to recombination active defects in block-cast multicrystalline silicon solar cells
}

\author{
Wolfram Kwapil, ${ }^{1, a)}$ Martin Kasemann, ${ }^{2}$ Paul Gundel, ${ }^{1}$ Martin C. Schubert, ${ }^{1}$ \\ Wilhelm Warta, ${ }^{1}$ Paula Bronsveld, ${ }^{3}$ and Gianluca Coletti ${ }^{3}$ \\ ${ }_{1}^{1}$ Fraunhofer Institute for Solar Energy Systems (ISE), Heidenhofstr. 2, 79110 Freiburg, Germany \\ ${ }^{2}$ Material Research Center, University of Freiburg, Stefan-Meier-Str. 21, 79104 Freiburg, Germany \\ ${ }^{3}$ Energy Research Centre of the Netherlands (ECN), Westerduinweg 3, NL-1755 LE Petten, The Netherlands
}

(Received 30 June 2009; accepted 12 August 2009; published online 25 September 2009)

\begin{abstract}
Solar cells in modules are reverse biased when they are shaded. This can lead to diode breakdown and eventually to the occurrence of hot spots, which may, in the extreme case, destroy the module by thermal degradation. We observed at least three different types of diode breakdown in multicrystalline silicon solar cells. One of them is found to be related to the recombination activity of defects. This type is indicated by a slow increase in the reverse current with reverse bias and a relatively low breakdown voltage around $-10 \mathrm{~V}$. The local breakdown voltage depends significantly on the level of contamination of the material. When the solar cell is reverse biased, the breakdown sites emit bright light which shows a broad spectral distribution in the visible range with a maximum at $700 \mathrm{~nm}$. (C) 2009 American Institute of Physics. [doi:10.1063/1.3224908]
\end{abstract}

\section{INTRODUCTION}

The diode breakdown behavior of silicon $p n$ junctions has been extensively studied in the 1950s and 1960s. Various physical mechanisms have been identified, the most important being avalanche breakdown, ${ }^{1}$ internal field emission (tunneling), ${ }^{2}$ and thermal breakdown. ${ }^{3}$ Avalanche breakdown occurs if the acceleration of electrons due to the electric field in reverse biased $p n$ junctions is sufficient for impact ionization in the space charge layer. ${ }^{1}$ Internal field emission is attributed to quantum mechanical tunneling of electrons from the valence band to the conduction band. The tunneling probability, however, is considerable only in highly doped step junctions. ${ }^{2}$ Thermal breakdown is also known as second breakdown since it can follow after avalanche breakdown, leading to strong heating of the silicon lattice. ${ }^{3}$

Light emission in the visible spectral region has been reported for all three breakdown phenomena. ${ }^{2,4}$ While a weak, red glow uniformly distributed over the surface was observed for silicon diodes in internal field emission, ${ }^{2}$ avalanche and thermal breakdown are known to emit bright light of white or yellow color in some selected spots. ${ }^{1,3}$

In this contribution we report on our observations of light emission in single small spots in reversely biased silicon solar cells. We present in the following a short summary of prior publications about avalanche breakdown in silicon $p n$ junctions. Due to the dominance of monocrystalline silicon in semiconductor industry, most of the older publications only deal with single crystals.

The breakdown voltage $V_{B}$ basically depends on the dopant concentrations of the emitter and base and may vary over a wide voltage range. ${ }^{5}$ The onset of breakdown is often accompanied by microplasma noise, i.e., it comes along with reverse current instability. ${ }^{6}$ Increasing the reverse bias, the

\footnotetext{
${ }^{a)}$ Electronic mail: wolfram.kwapil@ise.fraunhofer.de. Tel.: +49 7614588 5482 .
}

avalanche breakdown becomes stable and the reverse current increases exponentially. By measuring the light emission intensity of a set of microplasmas in dependence of the reverse current and then repeating the experiment while the microplasma spots were blackened out, it was found that most if not all of the leakage current is concentrated within the small microplasma channels and can be observed via the light emission. ${ }^{7}$ Most of the microplasmas are about 1-5 $\mu \mathrm{m}$ in diameter, ${ }^{8}$ leading to approximate reverse currents of $100 \mu \mathrm{A}$ per microplasma site. ${ }^{7}$ A strong correlation between the location of the microplasma spots and dislocations in the crystal was found. ${ }^{9}$ Another explanation of their origin involves precipitates in or close to the space charge layer, namely, $\mathrm{SiO}_{2}$ precipitates leading to electric field enhancement in their proximity. ${ }^{10}$ Moreover, Goetzberger and Shockley investigated the influence of intentional metal contamination of monocrystalline wafers on the breakdown behavior. They found that high impurity concentrations lead to a soft breakdown, whereas uncontaminated $p n$ junctions show hard breakdown behavior. ${ }^{11}$ By applying a potential probing technique, they observed that in their samples, the breakdown current often converged in one small spot which they assumed to be a metal precipitate. Here and in the following, the steepness of the breakdown with increasing voltage shall be characterized by the terms "soft" and "hard," a hard breakdown relating to a large increase in reverse current within a small increase in reverse bias as soon as the breakdown voltage has been reached.

In a number of publications measured spectra of avalanche breakdown were presented. ${ }^{4,12,13}$ The reported spectra mostly peak at around $620 \mathrm{~nm}$ and show a rather broad spectral distribution. It was noted, however, that the color varies depending on the depth of the space charge layer which was attributed to reabsorption of the emitted light. ${ }^{4}$ For the understanding of the broad spectral distribution, several physical mechanisms have to be taken into account. The most recent investigations postulate that bremsstrahlung in the 
Coulomb field of charged atoms as well as interband and intraband transitions involving interaction with phonons contribute to the spectrum. ${ }^{13}$

All the above mentioned results were obtained on small, monocrystalline silicon $p n$ junctions. The solar cells which are in use today, however, differ strongly from the samples which have been investigated in the past decades in several points.

(1) The industrial silicon solar cells are large in size (standard size being $15.6 \times 15.6 \mathrm{~cm}^{2}$ ).

(2) A considerable share of them is made of multicrystalline (mc) silicon. This leads to a high crystal defect density, i.e., grain boundaries and dislocations.

(3) They contain a relatively higher impurity concentration compared to the materials so far investigated. The detrimental impact of impurities on solar cell performance is governed by the transition metal impurity atoms. Most of them are precipitated at crystal defects.

(4) The surface is textured with structures of several micrometers depth in order to maximize light capture. Since the thin emitter follows closely the surface morphology, the electric field in the space charge layer is expected to vary laterally.

Because of all these differences, it is to be expected that breakdown in silicon solar cells is a more complex phenomenon than in a small single crystalline $p n$ junction.

This work is mainly motivated by two critical issues encountered in current industrial mc silicon solar cell production. First, the breakdown behavior is of paramount importance for the design of modern solar modules. A solar cell within a module string is reverse biased when shadowed. If breakdown occurs in highly localized spots, heating may be strong enough for the occurrence of so called "hot spots." They can eventually lead to the destruction of the module. Second, with the development of alternative routes to produce silicon feedstock (often termed upgraded metallurgical grade), a higher contamination level of the resulting wafers is expected. Although comparable cell efficiencies have been reached with this material on an industrial scale, ${ }^{14}$ a significantly reduced breakdown voltage compared to standard solar cells was reported, forcing the module manufacturers to adapt the module design ${ }^{14}$ associated with cost increase. Obviously, there is a correlation between contamination level of the wafer and the global breakdown. This contribution is intended to shed light on the local correlation between contamination and breakdown behavior.

\section{EXPERIMENTAL}

For our investigations, we used three different sets of samples. First, we characterized a solar cell which was made of high-quality mc silicon. It was processed in a standard industrial screen printing process including a wet chemical acidic etch. Second, wafers from five different mc silicon ingots were taken from different positions distributed over the ingot height and processed to solar cells. The ingots were made of high purity solar grade silicon feedstock with transition metals added to the silicon melt. The following blocks were cast:

(1) "Ni" contaminated with 40 ppmwt of nickel,

(2) "Cr-Fe-Ni" contaminated with a mixture of $40 \mathrm{ppmwt}$ chromium, 200 ppmwt iron, and 40 ppmwt nickel,

(3) "Fe" contaminated with 200 ppmwt iron, and

(4) "Cr"contaminated with 40 ppmwt chromium.

(5) In addition, "Ref," a reference ingot made of high purity solar grade feedstock was taken.

After crystallization, ingot cutting, and wafer slicing, the wafers were processed in one run in a standard industrial screen printing process including an acidic texturization step. The ingot characterization and the solar cell performance are reported in Refs. 15 and 16. It was verified with the help of neutron activation analysis that the ingots were contaminated as intended ${ }^{15}$ and impurity segregation toward the top of the ingot took place. In addition, a group of mc silicon solar cells was processed in a standard screen printing process starting with wafers which were damage etched. In this wet chemical process, rough surface inhomogeneities (e.g., saw damage) are smoothed, resulting in a shiny surface appearance. Thus, the influence of surface morphology effects on the microscopic investigations is mitigated.

For the characterization of the breakdown behavior we used the fact that breakdown in silicon causes light emission detectable by silicon charge coupled device cameras. In an electroluminescence (EL) setup, we increased the reverse voltage slowly, taking an EL image at each step. We limited our measurements to a maximum reverse current of $-2 \mathrm{~A}$ in order to avoid damage to the solar cells. We measured (i) the local heat development and reverse current density, (ii) the local recombination activity, and (iii) the local dislocation luminescence intensity with spatially resolved infrared imaging methods.

(i) The signal measured with the help of dark lock-in thermography ${ }^{17}$ (DLIT) is proportional to the local heat development. Assuming a constant reverse voltage distribution across the solar cell, ${ }^{18}$ DLIT can be used to image the local reverse current density across the $p n$ junction.

(ii) Qualitative information on the local recombination activity in the cell was obtained by EL imaging. ${ }^{19}$ There, the luminescence signal emitted from the base of the forward-biased silicon solar cell is detected by a camera with a silicon detector. The luminescence signal is related to the local junction voltage and to the local depth profile of the carrier density which is determined by the local recombination activity. At the low external voltages used for this measurement, the junction voltage is almost constant across the cell. In addition, the luminescence signal is influenced by the local wafer surface morphology. However, the existing luminescence intensity variations are dominated by the recombination activity in the material, especially the sharp features of low intensity that are discussed later. A low band-to-band luminescence signal 

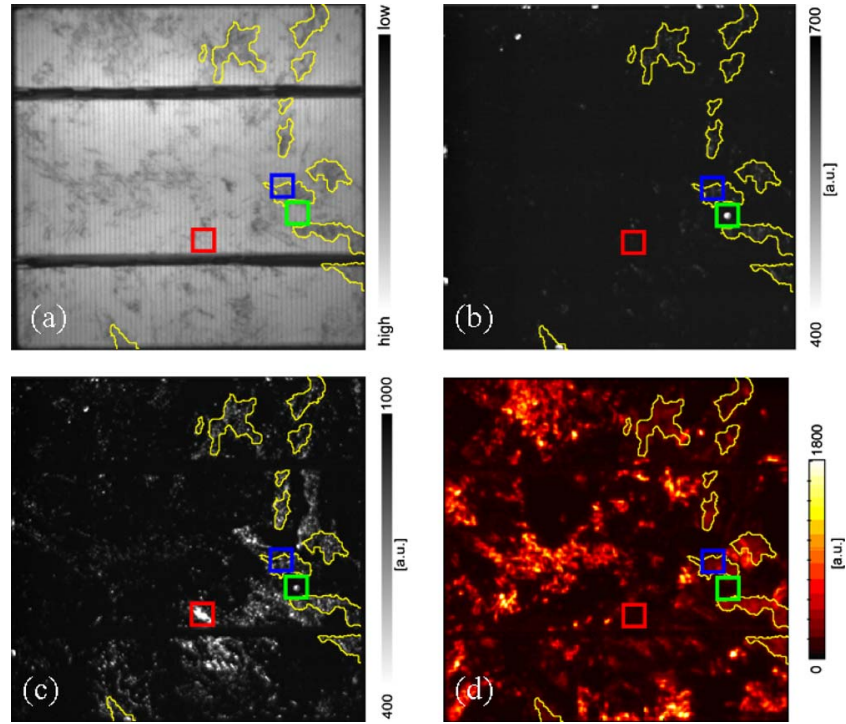

FIG. 1. (Color online) EL images of an industrial mc silicon solar cell in (a) forward bias $(600 \mathrm{mV}),(\mathrm{b})-12 \mathrm{~V}$, and (c) $-14 \mathrm{~V}$ reverse bias. The dislocation luminescence (d) - taken at $600 \mathrm{mV}$ forward bias-anticorrelates with regions of type II breakdown. A selection of prominent type II breakdown regions is marked with the yellow boundaries. Most other bright breakdown sites in image (c) are either of type I or type III. Weak breakdown light emission is mainly found in regions with high dislocation luminescence intensity. The colored rectangles indicate the position of the spots plotted in Fig. 2.

corresponds to a high recombination activity and thus to a high defect density.

(iii) Dislocation-related EL at room temperature was detected by an InGaAs-based camera through a narrow optical band-pass filter with a center wavelength at $1555 \mathrm{~nm}$. A clear correlation between the presence of dislocations and characteristic luminescence in this wavelength range has been found by several research groups. $^{20-23}$

For the microscopic investigation, we used EL/ photoluminescence (PL) spectroscopy mapping with a high resolution in the order of $1 \mu \mathrm{m}$. The setup comprises a confocal optical microscope and a sample holder attached to a piezostage. A laser beam $(532 \mathrm{~nm})$ can be focused onto the sample which generates electron-hole pairs in the silicon bulk. At this wavelength, the penetration depth of the laser beam is approximately $1 \mu \mathrm{m}$. The emitted luminescence signal is coupled into a glass fiber via the microscope optics and is directed to an IR and an UV/visible spectrometer. Similar to the EL intensity, the measured PL intensity indicates the local recombination activity. Alternatively, the sample can be connected to a voltage generator which allows applying a bias to the sample and thus generates EL or breakdown light emission.

\section{MEASUREMENTS}

\section{A. Coexistence of several breakdown types in one solar cell}

In Figs. 1(a)-1(c), an example of EL measurements in forward and reverse biases on an industrial solar cell made of

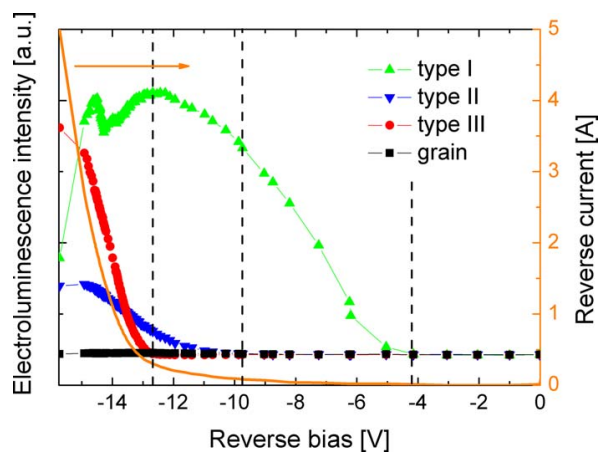

FIG. 2. (Color online) EL intensity vs reverse bias of four representative spots (position marked in Fig. 1) and global current characteristics (orange continuous line). The onset of the three types is tentatively marked by the dashed lines.

high-quality mc silicon is shown. As expected, bright spots start to appear at around $-5 \mathrm{~V}$, increasing in size and number when the reverse voltage is raised.

We could observe at least three different types of diode breakdown. ${ }^{24}$ These types differ in the voltage threshold at which they begin to break down as well as in the properties of the breakdown sites. It turns out that most of the breakdown spots on a solar cell can be classified according to the three different types (in the following termed types I, II, and III). The bias-dependent EL intensity of one representative spot of each type is shown in Fig. 2.

On this solar cell, type I occurs at very low reverse bias around $-5 \mathrm{~V}$. The dots are mostly located at the edge of the solar cell. They are very bright in appearance throughout most of the voltage range investigated. However, in most cases the heat development measured with the help of DLIT remains low. We often observed (see, e.g., also Fig. 4, region 3) that the EL intensity of type I breakdown sites drops abruptly at a certain reverse voltage (sometimes at several voltages). Possible mechanisms are, e.g., (i) the onset of additional breakdown sites in the vicinity induces a slight variation in the current transport due to Joule heating of the bus bars and the fingers of the front metallization (which connects the breakdown sites under observation) and therefore a change in the series resistance or (ii) additional breakdown paths reduce the local voltage available at the spot under consideration.

For type II, breakdown happens at a reverse bias around $-8.5 \mathrm{~V}$. These breakdown sites correlate well with recombination active areas. Both their breakdown light emission and the heat developed in these regions increase only slightly with increasing reverse bias, indicating a soft breakdown behavior. We found ${ }^{25}$ that these breakdown regions of increased defect density tend to show only weak dislocation luminescence [see Fig. 1(d) marked in yellow; values below 1000 counts]. On the contrary, defect regions with strong dislocation luminescence (values around 1800 counts) tend to generate only very small local reverse current at the reverse voltages used here. Roughly speaking, there is "no" relevant breakdown in regions of high dislocation luminescence intensity.

Type III is characterized by very high breakdown voltages around $-12.5 \mathrm{~V}$ and higher. These spots do not corre- 

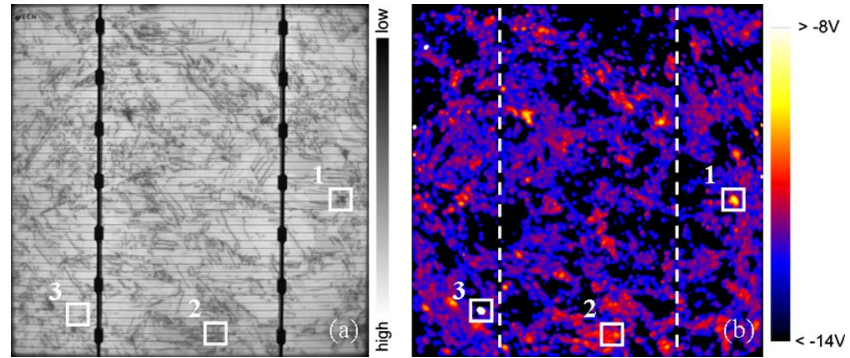

FIG. 3. (Color online) Reference solar cell taken at 55\% block height. (a) EL image in forward bias and (b) map of the local breakdown voltage (the position of the bus bars is shown by the dotted white lines). The white rectangles indicate the position of the spots plotted in Fig. 4.

late with regions of decreased carrier lifetime and grow brighter very quickly (hard breakdown). The global IV curve (orange line in Fig. 2) at high reverse bias is governed by the current transport through these regions, leading to large heat dissipation.

Bauer et al. ${ }^{26}$ found indications that type III breakdown happens at dislocations which had been selectively etched during the acidic texture etching process. The observed reduction in the breakdown voltage corresponds well to Sze's theory of avalanche breakdown due to electric field enhancement (electrostatic tip effect) at the bottom of etch pit cones with a small tip curvature. ${ }^{5} \mathrm{~A}$ further indication for the avalanche mechanism being the physical reason is the negative temperature coefficient of the reverse current ${ }^{27}$ which the authors measured in the respective breakdown region. ${ }^{26}$ Apart from the fact that it remains to be clarified why the preferential deep etching occurs at certain dislocations (which at the same time do not seem to be recombination active) while others are not affected, a reasonable explanation for the mechanism of type III breakdown has therefore been found.

\section{B. Breakdown voltage distribution on solar cells made of differently contaminated block- cast me silicon}

Since type III breakdown appears to be essentially process induced, it may be suppressed by modifying the wet chemical texturization step. ${ }^{26}$ In our experience, it actually depends very strongly on the cell process whether a solar cell exhibits hard type III breakdown or not. In addition, different mc material (with different saw damage) can react very differently to the same wet chemical texturization step. The breakdown labeled type II, however, relates to the silicon wafer material itself. In contrast to breakdown due to deep etch pits (type III), type II breakdown happens on every mc silicon solar cell. Especially under the aspect that there are ongoing efforts to reduce cost and energy consumption by developing new routes for feedstock production, it is worth investigating the implications of material properties on this type of breakdown behavior.

Figure 3 shows an example for the measurements taken from blocks 1 to 5. For the evaluation of the local breakdown voltage (b), an intensity threshold was defined in the EL measurements in order to distinguish between breakdown spots and background noise. Breakdown is detected when the

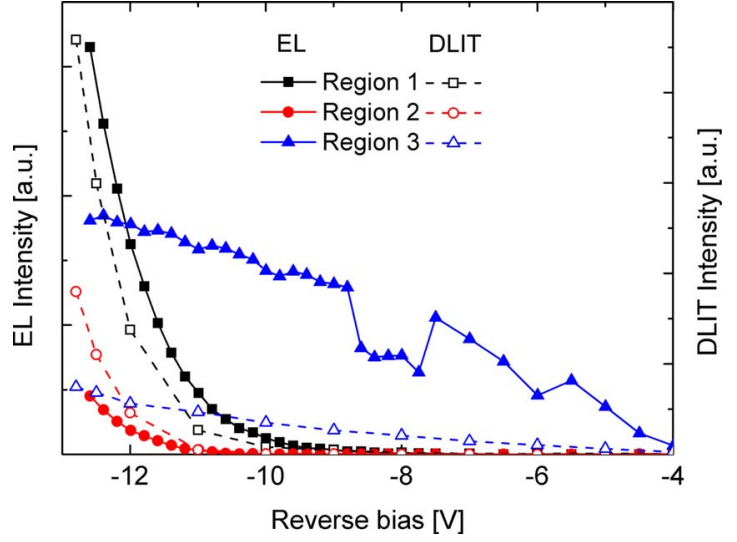

FIG. 4. (Color online) EL intensity (closed symbols) and DLIT intensity (open symbols) of three representative spots [marked in Fig. 3(b)].

intensity is larger than $\langle I\rangle+3 \sigma$, $\langle I\rangle$ being the average background noise level of a dark image and $\sigma$ denoting its standard deviation. We therefore systematically overestimate the local breakdown voltage; the weaker the local breakdown is, the larger the systematic error is. However, we estimate this error in the local breakdown voltage to be less than $10 \%$ for the weak breakdown sites. Moreover, due to refraction and reflection at the textured surface, the spots appear larger (some tens to some hundreds of micrometers) to the EL camera than they are in reality (below $5 \mu \mathrm{m}$ in diameter, see Fig. 6). Therefore, the diameter of the breakdown spots is systematically overestimated. Both error sources should nevertheless allow for comparison between the solar cells since the same procedure is applied to each measurement.

Almost all breakdown sites, represented by the local breakdown voltage map in Fig. 3(b), correlate well with the dark areas in the EL image [Fig. 3(a)] which signify recombination active defects. Only a few sites with early breakdown [type I, breakdown voltage $>-8 \mathrm{~V}$ in Fig. 3(b)] were found on each solar cell; they are not related to the recombination activity. We did not observe any hard breakdown due to deep etching (type III) in the voltage and current range up to $-2 \mathrm{~A}$. Therefore, the following analysis involves mainly type II breakdown.

The local breakdown voltage of the breakdown sites related to recombination active defects varies laterally. By comparing Figs. 3(a) and 3(b), it can be seen that breakdown regions which break down around $-9 \mathrm{~V}$ appear darker in the EL image in forward bias than regions that break down at higher reverse bias. The varying breakdown voltage results in different local bias-dependent measurement signals that are shown in Fig. 4. The EL as well as the DLIT intensity (which is proportional to the local current density) of representative spots of two regions with different breakdown voltages (labeled regions 1 and 2, respectively) are plotted versus the reverse bias. In addition, a typical early breakdown spot of type I is shown (region 3). The breakdown site in region 1, which sets on at lower reverse bias than the breakdown spot in region 2, grows brighter more quickly. This is also reflected in the heat development which is measured with DLIT. Therefore, at larger reverse bias, most of the reverse current is transported across the type II breakdown regions 


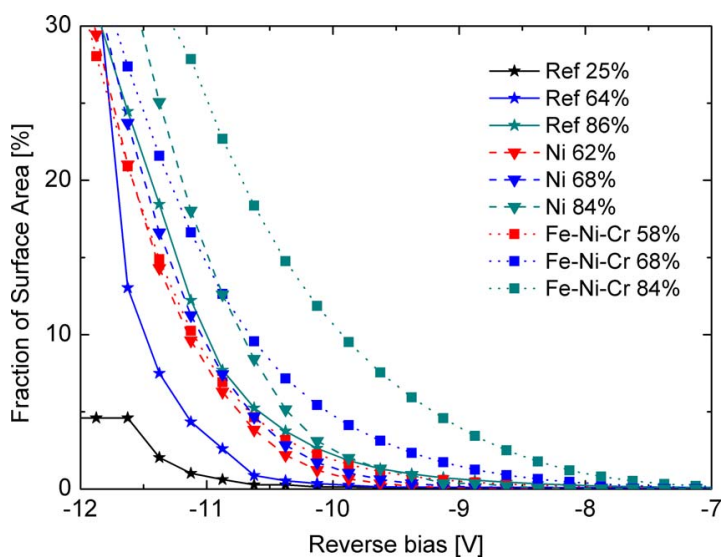

FIG. 5. (Color online) Fraction of the surface area which shows light emission due to breakdown. This graph shows measurements made on solar cells which were taken from three different ingots and different ingot heights (denoted by the percentage, $0 \%=$ bottom, $100 \%=$ top of the ingot).

which break down relatively early. Since the DLIT signal of type I breakdown sites remains low throughout the investigated voltage range (e.g., in region 3 ), they contribute only little to the breakdown current transport.

We found that the first onset of type II breakdown as well as the variation in local breakdown voltage depend strongly on the contamination level of the silicon wafer. Figure 5 shows the fraction of the surface area which emits light due to breakdown plotted against the reverse voltage. This is a measure for both the onset of type II breakdown in each solar cell and the lateral extension of the breakdown. The evaluation was performed in a similar way as the analysis of the local breakdown voltage.

In summary, two trends can be observed. First, the more contaminated the ingot is, the lower the local breakdown voltage is. Second, solar cells made of wafers, which were taken from close to the top of the ingot, possess a lower breakdown voltage than the wafers taken from the bottom or the center of the same ingot.

For example, we observe that type II breakdown begins last in the solar cells made of high purity solar grade feedstock. By comparing the solar cells from around $65 \%$ of the ingot height of the three different ingots, we find that the difference between the breakdown voltage of the reference block and the Ni contaminated ingot is about $1 \mathrm{~V}$, while the difference between the reference ingot and the $\mathrm{Fe}-\mathrm{Ni}-\mathrm{Cr}$ ingot is already about $2 \mathrm{~V}$. In this example, the breakdown voltage is decreased by about $20 \%$ due to ingot contamination.

An equally strong influence on breakdown voltage comes from the wafer position in the ingot. Solar cells from $25 \%$ block height of the reference ingot show first bright spots around $-10.0 \mathrm{~V}$, while the topmost ( $86 \%$ ingot height) reference solar cell already starts to break down below -9.0 $\mathrm{V}$. In the highly contaminated solar cells $(\mathrm{Fe}-\mathrm{Ni}-\mathrm{Cr})$, the onset of breakdown decreases significantly from around $-9.0 \mathrm{~V}$ (center of the ingot) to around $-7.5 \mathrm{~V}$ (84\% ingot height). Ingot position thus affects all samples regardless of their intentionally added contamination. For instance, the breakdown voltage at around $85 \%$ ingot height is about $10 \%$
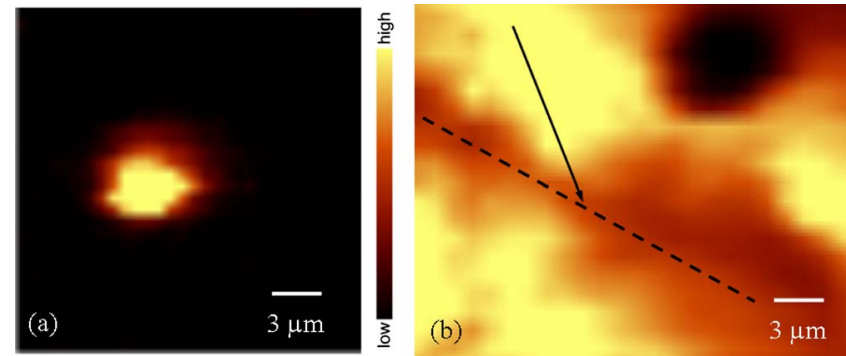

FIG. 6. (Color online) (a) High resolution EL map of the light emission in reverse bias at $-14 \mathrm{~V}$. (b) PL map of the same area. The arrow points on the site of the highest light emission intensity in reverse bias which is located at a grain boundary (marked by the dashed line). The dark dot in the upper right part is a particle used for orientation. The intensity scale applies to both images.

lower than the breakdown voltage around $65 \%$ ingot height for all three ingots. If the segregation of metal impurities during the crystallization is taken into account, the effect of ingot height on breakdown voltage can be explained by the influence of contamination too.

The evaluation of the slopes of the measured curves in Fig. 5 shows that contamination also affects the lateral breakdown voltage distribution. For the highly contaminated solar cells $(\mathrm{Fe}-\mathrm{Ni}-\mathrm{Cr})$, slowly increasing the reverse bias results in only a gradual increase in the breakdown surface fraction. By contrast, the steep slopes observed for the reference solar cells indicate that here breakdown happens within a rather small voltage range in many spots at once. The correlation between a slow increase in the breakdown surface fraction with reverse bias and the contamination of the solar cell is significant.

In addition to the results presented in Fig. 5, we performed the same measurements on the ingots contaminated solely with iron and chromium, respectively. Regarding the diode breakdown behavior, no significant difference between the several species of contamination was found. The fact that impurity atoms are present in a certain concentration seems to suffice for the development of a lower breakdown voltage. Note that all the investigated impurity species have in common that they possess a high diffusivity which enables them to travel to dislocation lines and grain boundaries and form precipitates within a relatively short time.

\section{Microscope investigations of type II breakdown sites}

In order to substantiate the obvious macroscopic link between recombination activity and local breakdown, we performed similar luminescence measurements on a microscopic level.

For these investigations, we used damage etched mc solar cells which were processed in a standard industrial screen printing process. Their behavior in reverse bias was characterized by macroscopic EL imaging, and sites showing a breakdown behavior classified as type II were selected for the following investigations.

A typical representative of these measurements is shown in Fig. 6. At a reverse bias of $-14 \mathrm{~V}$, the light generated in the breakdown sites is emitted in a spot of approximately 


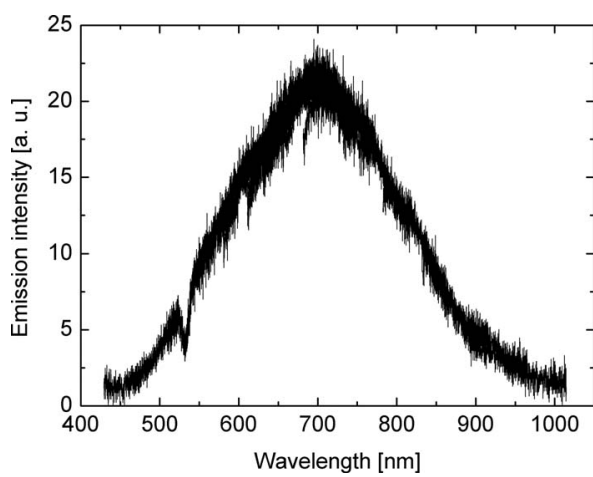

FIG. 7. Typical spectrum measured at a breakdown site of type II. The dip around $530 \mathrm{~nm}$ is caused by a filter.

3-5 $\mu \mathrm{m}$ in diameter, see Fig. 6(a). The spectrum, shown in Fig. 7 , is distributed over a broad wavelength range. Its maximum is found around $700-720 \mathrm{~nm}$. Compared to spectra obtained on monocrystalline silicon, ${ }^{4}$ this peak is shifted about $100 \mathrm{~nm}$ to the red end of the spectrum. Chynoweth and McKay observed that the color of the light depends on the distance between the sample surface and the $p n$ junction due to reabsorption. ${ }^{4}$ Since the $p n$ junction of our solar cells is located at a depth of around $0.5 \mu \mathrm{m}$, which is closer to the surface than most of the pn junctions investigated in Ref. 4, reabsorption in the silicon can probably be excluded.

Figure 6(b) shows the corresponding band-to-band PL map taken at a wavelength of $1100 \mathrm{~nm}$ at the breakdown site. The center of the breakdown intensity is located in the recombination active region marked by the black arrow. It is found within a grain boundary running along the dashed line. We confirmed with the help of a SEM (secondary electron detector) that the breakdown is not caused by an enhanced electric field at the tip of a deep etching at the grain boundary since the surface morphology does not contain any deep etch pits.

We conclude from the good spatial correlation that type II breakdown happens indeed at recombination active sites.

\section{DISCUSSION}

No physical model has been presented up to now for the occurrence of weak breakdown at recombination active defects. In our opinion, such a model should be able to explain the following findings which have been collected within this work.

(1) Recombination active defects at which type II breakdown occurs exhibit only weak dislocation luminescence. Vice versa, defects showing a strong dislocation luminescence hardly break down in the same voltage range as defects without such luminescence.

(2) The local breakdown voltage depends on the local recombination activity. Early type II breakdown is found in darker regions in forward bias EL images.

(3) The more the solar cell is contaminated with transition metal impurities, the earlier type II breakdown sets on. Unfortunately, currently we cannot substantiate this statement further because existing methods for the measurement of the local recombination activity can discern only between defects with relatively light impurity decoration. Above a certain decoration level, the measured recombination activity of the defect stays the same. In this case, the recombination activity is limited by the carrier diffusion toward the defect. Therefore, we can directly correlate the strength of the local recombination activity with the local breakdown voltage only in a limited range.

(4) Type II breakdown happens in singular spots of some micrometers in diameter. Although they are always found at recombination active defects, the reverse is not true: not all recombination active defects (which do not exhibit dislocation luminescence) break down in the investigated voltage range.

A qualitative explanation of some of these observations can be made based on a model that Kveder et al. ${ }^{21,22} \mathrm{em}$ ployed to successfully explain different experimental observations related to the recombination at clean and decorated dislocation networks. Following their argumentation, dislocation luminescence is generated by radiative recombination between energy levels (or bands) which are located close to the conduction band and the valence band, respectively. High dislocation luminescence intensity means that the radiative recombination between these defect levels is very strong. Weak dislocation luminescence is then explained by a suppression of radiative recombination by competing nonradiative recombination via additional defect levels closer to the center of the band gap. If precipitates are responsible for the formation of such deep defect levels, the soft breakdown in these regions could be attributed to the presence of precipitates in or close to the space charge layer. ${ }^{11}$ The occurrence of these precipitates increases with increasing overall metal contamination level.

Since type II breakdown is located only at some sites of recombination active defects, these must possess specific properties which distinguish them from others. We suggest several possible scenarios.

First, it is probable that the coincidence of both recombination activity at dislocations and grain boundaries and the roughness of the surface of either chemically polished or acidic etch-textured samples, which happens to be more pronounced at crystal defects (since the surface is not mechanically polished), is responsible for the local breakdown behavior. Second, as discussed in the previous paragraph, precipitates are likely to be present. The precipitate distribution and size along each dislocation line and their position relative to the space charge region (where eventually breakdown occurs) should then have a significant influence. In general, a higher contamination level should lead to more and larger precipitates. Then, if a certain constellation of precipitate with regard to the space charge layer is necessary for the onset of breakdown, this constellation becomes more probable to occur in contaminated silicon. Furthermore, local inhomogeneities in the emitter profile, possibly influenced by the presence of precipitates, may also play a role.

All these effects have in common that they are likely to lead to a variation in the local electric field strength. Since all known breakdown mechanisms involve charge carriers 
within a strong electric field, fundamentally the local increase in the electric field induced by one of the above or a further local defect constellation is expected to result in the laterally varying breakdown behavior.

\section{CONCLUSION}

We have shown that diode breakdown in mc silicon solar cells can be classified according to three distinctly different types regarding light emission versus reverse voltage. The breakdown of type II is related to recombination active crystal defects. No breakdown is found at defects which show a strong dislocation luminescence. The onset of type II breakdown is clearly correlated with the degree of contamination of the feedstock material. A higher intentionally added impurity content leads to lower local breakdown voltage irrespective of the type of the metal contaminants investigated. This is also true for the local breakdown in different regions within one solar cell. We measured differences in breakdown voltage on five differently contaminated silicon materials of $0.5 \mathrm{~V}$ to more than $2 \mathrm{~V}$ at the same ingot height. Moreover, local breakdown sets on earlier for wafers which come from a position closer to the top of the ingot. Since metal impurity content increases due to segregation toward the top, this again confirms the correlation of type II breakdown with impurity content.

Also microscopically (with a resolution in the order of $1 \mu \mathrm{m})$ we observed that the breakdown of type II happens at positions of recombination active crystal defects. The breakdown spots are approximately $3-5 \mu \mathrm{m}$ in diameter. Their spectrum emitted during breakdown is broadly distributed with a maximum around $700 \mathrm{~nm}$.

We suggested different possible scenarios which might lead to type II breakdown. However, whether one or several of them constitute the underlying physical mechanism of type II breakdown needs yet to be determined.

\section{ACKNOWLEDGMENTS}

The authors would like to thank Adolf Goetzberger as well as Otwin Breitenstein, Jan Bauer, and Jan-Martin Wagner for fruitful discussions. This work was supported by the German Federal Ministry for the Environment, Nature Conservation and Nuclear Safety within the research cluster "SolarFocus" (Contract No. 0327650E) and the European "CrystalClear Integrated Project" (Contract No. SES6-CT_2003502583).
W. Mönch, Phys. Status Solidi 36, 9 (1969).

${ }^{2}$ A. G. Chynoweth and K. G. McKay, Phys. Rev. 106, 418 (1957).

${ }^{3}$ J. Tauc and A. Abrahám, Phys. Rev. 108, 936 (1957).

${ }^{4}$ A. G. Chynoweth and K. G. McKay, Phys. Rev. 102, 369 (1956).

${ }^{5}$ S. M. Sze, Physics of Semiconductor Devices, 2nd ed. (Wiley, New York, 1981).

${ }^{6}$ D. J. Rose, Phys. Rev. 105, 413 (1957); R. J. McIntyre, J. Appl. Phys. 32, 983 (1961)

${ }^{7}$ A. G. Chynoweth and K. G. McKay, J. Appl. Phys. 30, 1811 (1959).

${ }^{8}$ R. H. Haitz, A. Goetzberger, R. M. Scarlett, and W. Shockley, J. Appl. Phys. 34, 1581 (1963).

${ }^{9}$ A. G. Chynoweth and G. L. Pearson, J. Appl. Phys. 29, 1103 (1958).

${ }^{10}$ W. Shockley, Solid-State Electron. 2, 35 (1961).

${ }^{11}$ A. Goetzberger and W. Shockley, J. Appl. Phys. 31, 1821 (1960).

${ }^{12}$ R. Newman, Phys. Rev. 100, 700 (1955).

${ }^{13}$ N. Akil, S. E. Kerns, D. V. Kerns, Jr., A. Hoffmann, and J.-P. Charles, IEEE Trans. Electron Devices 46, 1022 (1999).

${ }^{14}$ V. Hoffmann, K. Petter, J. Djordjevic-Reiss, E. Enebakk, J. T. Håkedal, R. Tronstad, T. Vlasenko, I. Buchovskaja, S. Beringov, and M. Bauer, in Proceedings of the 23rd European Photovoltaic Solar Energy Conference, Valencia, Spain, 2008 (unpublished)

${ }^{15}$ G. Coletti, P. C. P. Bronsveld, R. Kvande, L. J. Geerligs, and C. C. Swanson, presented at the Workshop on Solar Grade Feedstock Specifications, Amsterdam, The Netherlands, 2008 (unpublished).

${ }^{16}$ G. Coletti, P. Bronsveld, R. Kvande, L. J. Geerligs, L. Arnberg, H. Habenicht, W. Warta, and C. Knopf, in Proceedings of the 18th International Photovoltaic Science and Engineering Conference, Kolkata, India, 2009 (unpublished).

${ }^{17}$ O. Breitenstein and M. Langenkamp, Lock-In Thermography-Basics and Use for Functional Diagnostics of Electronic Components (Springer, Berlin, 2003).

${ }^{18}$ I. E. Konovalov, O. Breitenstein, and K. Iwig, Sol. Energy Mater. Sol. Cells 48, 53 (1997).

${ }^{19}$ P. Würfel, T. Trupke, T. Puzzer, E. Schäffer, W. Warta, and S. W. Glunz, J. Appl. Phys. 101, 123110 (2007).

${ }^{20}$ M. A. Drozdov, A. A. Patrin, and V. T. Tkachev, JETP Lett. 23, 597 (1976).

${ }^{21}$ V. Kveder, M. Badylevich, W. Schröter, M. Seibt, E. Steinman, and A. Izotov, Phys. Status Solidi A 202, 901 (2005)

${ }^{22}$ V. V. Kveder, E. A. Steinman, S. A. Shevchenko, and H. G. Grimmeiss, Phys. Rev. B 51, 10520 (1995).

${ }^{23}$ M. Kittler, W. Seifert, T. Arguirov, I. Tarasov, and S. Ostapenko, Sol. Energy Mater. Sol. Cells 72, 465 (2002).

${ }^{24}$ W. Kwapil, M. Kasemann, J. Giesecke, B. Michl, and W. Warta, in Proceedings of the 23rd European Photovoltaic Solar Energy Conference, Valencia, Spain, 2008 (unpublished).

${ }^{25}$ M. Kasemann, W. Kwapil, M. C. Schubert, H. Habenicht, B. Walter, M. The, S. Kontermann, S. Rein, O. Breitenstein, J. Bauer, A. Lotnyk, B. Michl, H. Nagel, A. Schütt, J. Carstensen, H. Föll, T. Trupke, Y. Augarten, H. Kampwerth, R. A. Bardos, S. Pingel, J. Berghold, W. Warta, and S. W. Glunz, in Proceedings of the 33rd IEEE Photovoltaic Specialists Conference, San Diego, CA, 2008 (unpublished).

${ }^{26}$ J. Bauer, J.-M. Wagner, A. Lotnyk, H. Blumtritt, B. Lim, J. Schmidt, and O. Breitenstein, Phys. Status Solidi (RRL) 3, 40 (2009).

${ }^{27}$ O. Breitenstein, J. Bauer, A. Lotnyk, and J.-M. Wagner, Superlattices Microstruct. 45, 182 (2009). 